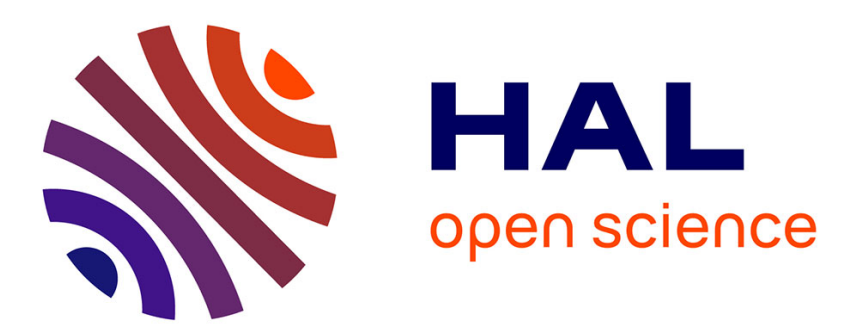

\title{
A Unifying Notification System To Scale Up Assistive Services
}

Charles Consel, Lucile Dupuy, Hélène Sauzéon

\section{To cite this version:}

Charles Consel, Lucile Dupuy, Hélène Sauzéon. A Unifying Notification System To Scale Up Assistive Services. ASSETS - The 17th International ACM SIGACCESS Conference on Computers and Accessibility, Oct 2015, Lisbon, Portugal. 10.1145/2700648.2809855 . hal-01225637

\section{HAL Id: hal-01225637 \\ https://hal.inria.fr/hal-01225637}

Submitted on 24 Nov 2015

HAL is a multi-disciplinary open access archive for the deposit and dissemination of scientific research documents, whether they are published or not. The documents may come from teaching and research institutions in France or abroad, or from public or private research centers.
L'archive ouverte pluridisciplinaire HAL, est destinée au dépôt et à la diffusion de documents scientifiques de niveau recherche, publiés ou non, émanant des établissements d'enseignement et de recherche français ou étrangers, des laboratoires publics ou privés. 


\section{A Unifying Notification System To Scale Up Assistive Services}

\author{
Charles Consel \\ Bordeaux INP / Inria \\ Talence, France \\ charles.consel@inria.fr
}

\author{
Lucile Dupuy \\ Inria \\ Talence, France \\ lucile.dupuy@inria.fr
}

\author{
Hélène Sauzéon \\ University of Bordeaux / Inria \\ Talence, France \\ helene.sauzeon@inria.fr
}

\begin{abstract}
Aging creates needs for assistive technology to support all activities of daily living (meal preparation, dressing, social participation, stove monitoring, etc.). These needs are mostly addressed by a silobased approach that requires a new assistive service (e.g., a reminder system, a pill prompter) to be acquired for every activity to be supported. In practice, these services manifest their silo-based nature in their user interactions, and more specifically, in the heterogeneity of their notification system. This heterogeneity incurs a cognitive cost that prevents scaling up assistive services and compromises adoption by older adults.

This paper presents an approach to scaling up the combination of technology-based, assistive services by proposing a unifying notification system. To do so, (1) we propose a decomposition of assistive services to expose their needs in notification; (2) we introduce a notification framework, allowing heterogeneous assistive services to homogeneously notify users; (3) we present how this notification framework is carried out in practice for an assisted living platform.

We successfully applied our approach to a range of existing and new assistive services. We used our notification framework to implement an assistive platform that combines a variety of assistive services. This platform has been deployed and used 24/7 at the home of 15 older adults for up to 6 months. This study provides empirical evidence of the effectiveness and learnability of the notification system of our platform, irrespective of the cognitive and sensory resources of the user. Additional results show that our assisted living platform achieved high user acceptance and satisfaction.
\end{abstract}

\section{Categories and Subject Descriptors}

K.4.2 [Computers and Society]: Social Issues-Assistive technologies for older adults; J.4 [Social and Behavioral Sciences]: Psychology

\section{General Terms}

Notification Systems, Field Study, Human Factors, Measurement, Performance

\section{Keywords}

Assisted living platform; Assistive technologies; Pervasive computing; Notification Systems; Field Study; Human Factors; Measurement; Performance

\section{INTRODUCTION}

Despite the many technological innovations available to assist older adults with cognitive decline in their daily life [31], their silobased nature makes it a challenge to aggregate them as the older adult requires more services to assist an increasing number of daily activities. ${ }^{1}$ A proliferation of technology-based services incurs an unrealistic cognitive load for the user, as documented in the literature on aging [34]. A key obstacle to scaling up technology-based assistive services is the heterogeneity of their user interfaces: because most existing services are developed using a silo-based approach, they impose their own specific interfaces. Older adults thus need to learn these interfaces, even though they often are not proficient with technology. The cognitive cost of this learning process may outweigh the potential benefits of the assistive device, preventing its adoption [22].

The range of services needed to assist older adults is so wide and diverse that attempting to unify their user interactions appears unrealistic. Yet, a close examination of existing services permits to outline a decomposition of their user interactions into two categories: notifications and task-specific interactions. In this context, notifications are issued by assistive services to provide the user with some information such as a situation and an event. As an example, consider a reminder system where notifications request the user's attention when an event has occurred, displaying the event information. These notifications can be made generic to cover, not only the reminder system, but a variety of assistive services, lowering their cognitive cost. Task-specific interactions are those mainly concerned with configuring the assistive service and feeding it with data, if necessary. For example, if the reminder system relies on a shared Web calendar, its network address needs to be defined at configuration time via task-specific interactions. As well, new events need to be entered in this service via task-specific interactions.

Reviewing existing assistive services [14] reveals that this decomposition can be applied to most of them. Furthermore, according to interviews we conducted with stakeholders, task-specific interactions are often performed by professional and family caregivers. For example, events are entered in a calendar by caregivers (doctor appointments, visit of a family member, etc.). In practice, our approach can accomodate existing assistive services by wrapping their notifications with a software layer, or be used as a basis to design new assistive services. In both cases, our approach provides guide-

\footnotetext{
${ }^{1}$ abledata.com
} 
lines to define notifications for assistive services. For the service developer, these guidelines take the form of an API.

This paper. We present an approach towards unifying interactions between the user and assistive services. This approach consists of a decomposition method that separates user interactions into taskspecification ones and notifications. Then, notifications are homogenized to facilitate the recurring usage of the assistive services. In practice, we provide an API to support notifications and to enforce their homogeneity. Besides the API, notifications are further homogenized by the use of a unique channel between the user and the assistive services. In the first implementation of our platform, this unique channel corresponds to a tablet. By enforcing the use of an API and unifying the presentation of notifications on a tablet, new assistive services can be introduced without incurring additional cognitive cost, aside from the task-specific interactions.

To validate our approach, we show how it successfully applies to a number of existing and new services that cover the three main domains of assisted living, namely, monitoring of activities of daily living (ADLs), safety and security, and social participation [27]. Specifically, our proposed API is implemented in an assisted living platform that has been deployed at the home of 15 older adults, aged 81 on average. Furthermore, we are conducting a field study, using our platform equipped with a variety of assistive services, at the home of older adults. Preliminary results show that our approach to scaling up the assistive services via a uniform notification system is effective and learnable, and achieves user acceptance and satisfaction. Finally, our field study allows us to go beyond a lab study: we put into practice our notification framework with assistive applications and test our implementation in the wild.

\section{RELATED WORK}

Cognitive cost of assistive services. Using assistive services incurs a cognitive cost for users. As showed by Lindenberger et al., an assistive service may not be adopted, if the resources (physical and cognitive) it requires, outweigh the payoff [22]. Beyond the cost of an assistive service, Sherer et al. recommend that a user with cognitive decline should not be introduced to more than three assistive tools, to prevent the older adult from being confused and overwhelmed [34].

These works identify the limitations to introducing services for assisted living purposes. They call for approaches to facilitating interactions with assistive services and lifting the limit on the number of services a declining older adult can cope with.

Design principles for older adults. Designing technology for older adults have been studied extensively by the HCI community. A comprehensive account is given by Fisk et al. [12]; it includes the characterization of older adult users, a presentation of design principles and guidelines, and examples illustrating the approach. As promoted by the authors, user-centered design should drive the development of assistive systems, taking into account the characteristics of aged-related declines in abilities.

Fisk et al. concentrate on the development of assistive tools from a unitary perspective. Not only does this perspective yield silobased technologies, but it does not address the issue of combining these technologies. As increasingly many assistive technologies are needed to address the difficulties of the aging user, a unifying approach is needed to scale up the assistance.

Notification systems. Most studies on notification systems investigate the costs, benefits and the optimal display of notifications in the context of desktop and mobile computing tasks [26]. Some works are centered on the human attention system and study the effects of notifications on ongoing computer tasks (e.g., [24]). To guide the evaluation of notification systems, McCrickard et al. propose a design model of user goals that is based on three critical parameters: interruption, reaction, and comprehension [25]. The design of our notification system addresses each of these parameters. Other works of notification systems examine notifications in the context of users with sensory impairments and assess the disruptiveness and effectiveness of notifications using multimodal interactions [38, 39]. User acceptance of notifications has also been explored; Vastenburg et al. conducted a user study of acceptance of notifications in a controlled home setting [36]. They show that user acceptance depends on whether the level of intrusiveness of a notification is related to its level of urgency. We use this key finding to structure our model of notifications.

Assisted living platforms. There is a range of platforms for assisted living aimed at older adults that have been developed for more than a decade, as surveyed by Chan et al. [6] and Rashidi and Mihailidis [31]. Most of these platforms are used in a setting where participants come to a research apartment to perform certain tasks (e.g., $[31,30,1])$. This setting makes it difficult to assess user acceptance and satisfaction of the proposed approaches because the user does not interact with the technology on a daily basis, over a period of time. Furthermore, older adults adopt routines to optimize their daily functioning at home [3]. This situation calls for field studies in a naturalistic setting to strengthen the evaluation of assisted living platforms.

Area-specific assistive services. The HCI community has proposed principles and guidelines to design assistive services that target specific needs of older adults. This includes family/peer interaction (e.g., PeerCare [32] and Message Center [41]), monitoring daily activities (e.g., Digital family portraits [33] and DigiSwitch [4]), and monitoring health-related activities (e.g., medication taking [20, 21, 17]).

Our work explores how a notification framework can contribute to scaling up the combination of silo-based assistive services across domains of assisted living, while achieving user acceptance and satisfaction.

\section{A UNIFYING FRAMEWORK FOR NO- TIFICATIONS}

This section presents our framework, which unifies notifications of assistive services. To do so, we first introduce how user interactions can be split into two groups: notifications and task-specific interactions. Second, we define a model for notifications that consists of two types of interactions, their interaction protocol, and their API. Third, we instantiate this model by showing how a tablet can be used to realize notifications. Last, we briefly present an assisted living platform that implements our proposed framework.

Throughout this section, we illustrate our approach with examples of assistive services taken from all domains of assisted living. Each step of our approach is instantiated with one of these examples. A selection of all the examples we have studied is presented in Figure 1.

\subsection{Decomposing Interactions}

Task-specific interactions mostly involve providing both configuration parameters and specific data to an assistive service. For assistive services that are networked, typical configuration parameters include network parameters, privacy requirements, and caregiver contact information. Let us examine our decomposition of interactions with respect to the main domains of assisted living. For the 


\begin{tabular}{|c|c|c|c|c|c|}
\hline Domain & Service & Description & $\begin{array}{l}\text { Task-specific in- } \\
\text { teractions }\end{array}$ & Notifications & Type \\
\hline \multirow[t]{2}{*}{ Safety } & $\begin{array}{l}\text { Activity } \\
\text { checker }\end{array}$ & $\begin{array}{l}\text { It checks whether the user gets out of bed } \\
\text { in the morning prior to a set time }\end{array}$ & Latest time & Alert & $\mathrm{C}$ \\
\hline & Pill prompter & It reminds the user to take medication & $\begin{array}{l}\text { New prescrip- } \\
\text { tion }\end{array}$ & $\begin{array}{l}\text { Reminder \& Pill } \\
\text { info }\end{array}$ & $\mathrm{C}$ \\
\hline \multirow[t]{2}{*}{ Security } & $\begin{array}{l}\text { Night door } \\
\text { monitoring }\end{array}$ & $\begin{array}{l}\text { It alerts the user if the entry door is open } \\
\text { at night. }\end{array}$ & $\begin{array}{l}\text { Night parame- } \\
\text { ters }\end{array}$ & Alert & $\mathrm{C}$ \\
\hline & Stove monitor & $\begin{array}{l}\text { It monitors the use of the stove and alerts } \\
\text { the user when the stove is left unattended } \\
\text { more than a set time }\end{array}$ & $\begin{array}{l}\text { Max. time unat- } \\
\text { tended }\end{array}$ & Alert & $\mathrm{C}$ \\
\hline \multirow[t]{2}{*}{ ADLs } & $\begin{array}{l}\text { ADL re- } \\
\text { minder }\end{array}$ & $\begin{array}{l}\text { It reminds the user to perform certain } \\
\text { ADLs }\end{array}$ & $\begin{array}{l}\text { ADLs of inter- } \\
\text { est and their } \\
\text { schedules }\end{array}$ & $\begin{array}{l}\text { Reminder } \quad \& \\
\text { ADL info }\end{array}$ & $\mathrm{NC}$ \\
\hline & $\begin{array}{l}\text { Bill manage- } \\
\text { ment }\end{array}$ & $\begin{array}{l}\text { It reminds the user of recurring bills to } \\
\text { be paid }\end{array}$ & Company name & $\begin{array}{ll}\text { Reminder } & \& \\
\text { Billing info } & \\
\end{array}$ & $\mathrm{NC}$ \\
\hline \multirow[t]{2}{*}{ Social participation } & email notifier & $\begin{array}{l}\text { It notifies the user for new email mes- } \\
\text { sages }\end{array}$ & $\begin{array}{l}\text { List of VIP } \\
\text { email addresses }\end{array}$ & $\begin{array}{l}\text { Notification } \\
\& \quad \text { message } \\
\text { excerpt }\end{array}$ & $\mathrm{NC}$ \\
\hline & $\begin{array}{l}\text { Municipal } \\
\text { events }\end{array}$ & $\begin{array}{l}\text { It announces social events for senior } \\
\text { adults, organized by their municipality }\end{array}$ & Preferences & $\begin{array}{l}\text { Notification } \quad \& \\
\text { event name }\end{array}$ & $\mathrm{NC}$ \\
\hline
\end{tabular}

'NC' and ' $C$ ' correspond to the type of notifications that is either non-critical or critical, respectively.

Figure 1: Our approach applied to examples of assistive services and target user profiles

domain of ADLs, consider the example of a pill prompter: an assistive service that reminds the user of medication to be taken. An example of a task-specific interaction for this service is to enable the caregiver to define/update a doctor's prescription. Another example is to customize the service by defining the snooze length for alerts. Notifications include issuing a reminder to take pills and an alert when the prescription is about to expire.

For the domain of safety and security, consider the example of a door monitor; this assistive service aims to prevent the entry door of a home from being left open and unattended. Task-specific interactions for this service includes its adaption to the user's needs and preferences. For example, the service may be parameterized with respect to the time during which the door can be open unattended, and the period during which door activities are considered to be daytime. Notifications consist of alerting the user when the door is left open.

For the domain of social participation, let us take the example of an email notifier. This assistive service notifies the user when (s)he receives email messages. Task-specific interactions for this service include setting the email account, parameterizing the occurrences of notifications (w.r.t. number of messages, specific senders, etc.). Notifications consist of informing the user of new message(s) (possibly displaying an excerpt from message(s)).

\subsection{A Model for Notifications}

Now that interactions have been classified as task specific and notifications, we need to further work on the notifications by introducing a model of interactions. This model is simple but realistic in that it has been applied to a range of existing and new assistive services (see Figure 1).

\subsubsection{Notification Types}

Field experiments show that user acceptance of notification systems depends on the level of urgency of the messages (e.g., [35]). To account for this key finding, we defined two categories of notifications: non-critical and critical. Non-critical notifications are the ones that do not need to be immediately attended to by the user; they announce a situation or an information. In contrast, critical interactions correspond to situations of assistance that may involve safety or security; the user's attention is thus requested. Determining whether a notification is non-critical or critical depends on a number of parameters, such as the nature of the assistive service, the target user, the user environment, etc. Not only do we introduce these types of notifications at a conceptual level, but we also provide mechanisms to ensure that this distinction is propagated throughout the development of an assistive service so as to deliver an appropriate level of intrusiveness.

A major benefit of the critical/non-critical dichotomy is that it allows to reduce the cognitive cost of both the interpretation and the decision making process relative to notifications. Such approach is highly recommended for older adults [22].

For our deployed assisted living platform, examples of non-critical notifications include an email notice, an event reminder, and a fridge door monitor. These assistive services do not depend on whether the user acknowledges their announcements. Critical interactions have been used for applications such as the entry door monitor, the stove monitor, and the activity monitor. In these examples, the absence of user input typically leads to eventually solicit a caregiver. More examples can be found in Figure 1.

\subsubsection{Interaction Protocols}

As reported by Vastenburg et al., user acceptance of notifications in the home can be improved by adjusting the level of intrusiveness of the presentation to the message urgency [36]. As a result, non-critical and critical notifications call for different interaction protocols. The protocol for non-critical interactions is that a situation or some information is announced. We propose that after a given time, if the user has not attended to the notification, it disappears and is assumed to be accumulated with other unattended non-critical notifications.

Critical notifications require user input because, by the definition given above, they involve safety or security. Consequently, when 
an assistive service issues a critical notification, we propose to give it a high level of intrusiveness to match the high value of the message [36]. Specifically, the notification will be repeated until the user responds. Furthermore, after a given time, the service will take emergency actions, such as turning off the stove and, in most cases, calling a caregiver.

\subsubsection{An API}

Our model is mapped into an API to enforce it at development time. In doing so, the programmer of an assistive service implements the service notifications following the notification types determined by the human factor expert for a given target user profile. Each user profile takes into account the dimensions relevant to the task to be assisted: the physical status, the functional status and the cognitive status. Typically, a door alert will trigger a non-critical notification for users with very good autonomy and a critical notification for lesser autonomous users. The collaboration between the programmer and the human factor expert is essential to ensure that the level of intrusiveness of the notification corresponds to the urgency of the situation and the user profile. In practice, our platform allows for different applications to be available for a given task (e.g., door monitor) thanks to an online catalog. As a result, users with different profiles will be given different assistive services. This approach contributes to achieving user acceptance. Figure 1 provides examples of notifications, whose types (rightmost column) are defined with respect to the user profiles of our user study.

Notice that our API abstracts over the way notifications are implemented. As will be discussed next, they may be carried out with a tablet. In principle, other devices could be used such as a TV or even a smart phone. We describe the main operations included in our API at a conceptual level, omitting the programming details.

A non-critical notification corresponds to an operation that takes a title, a notification content and an image. The title is used to categorize the notification in a pre-defined list (e.g., caution, reminder, FYI). The notification content corresponds to the message to be delivered to the user. An image can be used when dual coding (image and text) improves user performance to respond to the notifications of the application, as suggested by Warnock et al. [39]. Rendering the image depends on the functionality of the device used for interactions.

Because of its nature, a critical notification prompts the user to provide information that allows the application to take a course of action. Consequently, in addition to the input parameters of the non-critical notification, the critical one also takes a list of possible responses for the user to choose from. The user selects one of them to answer the question that corresponds to the notification message parameter.

Finally, it could be argued that our API prevents existing assistive services from being reused. In most cases, reuse can be achieved by wrapping an existing service with our API. This strategy is facilitated by the fact that assistive services are commonly implemented in the form of a Web service. In this case, we implement a software layer that operates the target Web service and calls our API whenever notifications need to be sent to the user.

\subsection{An Interaction Support: A Tablet}

We now instantiate our model of notifications with respect to a device, namely a tablet. Older adults have been shown to adapt well to touchscreen tablets [12] and to perform better than with the desktop and mouse [11]. As well, a key advantage of this device is that it can autonomously support interactions with a user, unlike a TV that requires a remote control for user input.

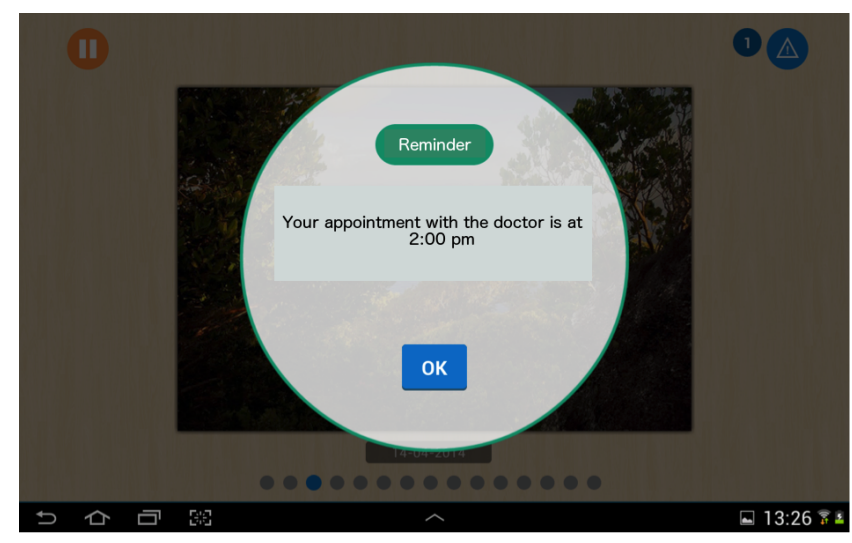

Figure 2: An example of a non-critical notification

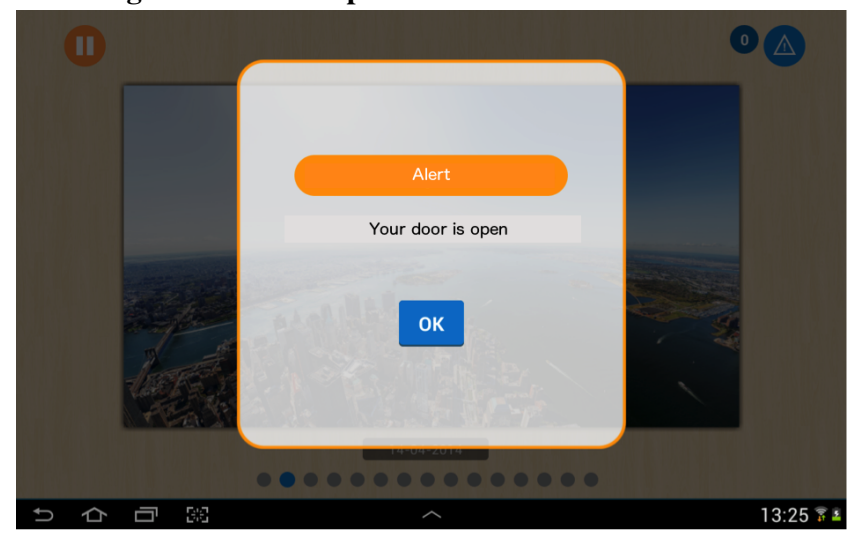

Figure 3: An example of a critical notification

To account for the capabilities of our target users, we strive to minimize the attentional resources required by assistive services. To do so, we increase multisensory integration to render notifications, making it easier to discriminate between notification types for older adults $[18,28]$. In practice, we leverage the features of the tablet to implement multi-modal coding of notifications, integrating tones, shapes, colors, and text. Let us now examine how our approach is carried out in practice and what benefits are brought to the user.

To allow the user to distinguish between non-critical and critical notifications, a specific tone was chosen for each type of notifications. As a result, users can quickly make the difference and do not bother going to the tablet when they hear a non-critical notification and are busy doing something else. To reduce the level of intrusiveness for non-critical notifications, our implementation uses a softer volume and tone for these notifications than for critical ones. By reducing the intrusiveness of these interruptions, we address the first of McCrickard et al.'s critical parameters of notification systems [25].

In addition to using different tones, we also use two different visual frames to display non-critical and critical notifications. Examples of notifications are displayed in Figures 2 and 3. As can be noticed, notifications are surrounded by a different shape, depending on their criticality: a green circle for a non-critical notification and an orange rectangle for a critical one. This strategy further reduces attentional resources and improves the ability for the user to distinguish between the two types of notifications, lowering the cognitive cost for making a decision. As such, this strategy improves the reaction to notifications using preattentive processing, thus addressing the second of McCrickard et al.'s critical parameters of 
notification systems [25]. Note that in the current implementation, images are not used for notifications.

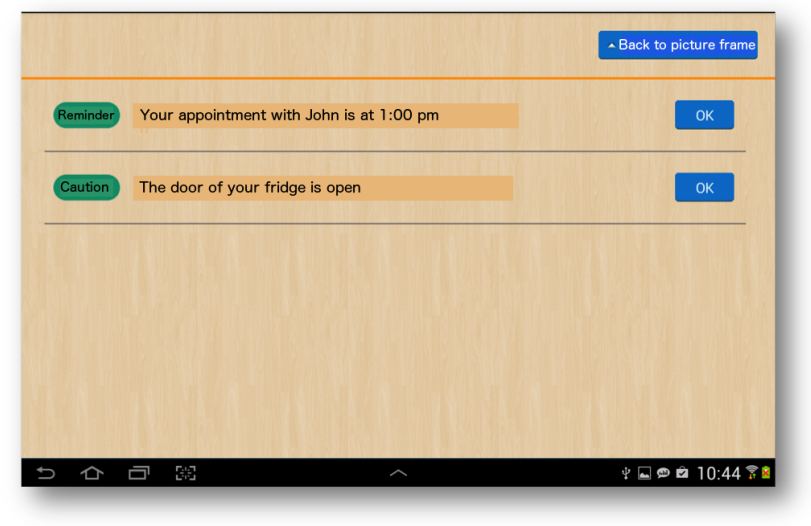

Figure 4: An example of a list of unattended notifications

Non-critical notifications can be attended at a later time by the user. In this case, they are gathered and are accessible by the user at their convenience. Unattended notifications are signaled to the user by the icon in the right corner of the tablet screen. This is illustrated in Figure 5 where the number of unattended notifications is displayed in a blue circle, at the top right corner of the screen. In Figure 4, we show a list of unattended notifications that the user can access by touching on the blue triangle. In contrast, a critical notification does not disappear from the screen and the tone is played periodically, until the notification is handled by the user.

Towards addressing the comprehension parameter of McCrickard et al.'s model [25], we keep messages short and located at the center of the notification window. To further explore this parameter, it needs to be considered in the context of assisted living. For critical notifications, the goal of the system is for the user to understand the message and resolve the safety-critical situation that triggered the notification. As explained earlier, the notification repeats until the message is understood and the situation resolved. For non-critical notifications, the message can be kept in the list of unattended notifications until the user decides that remembering the information is no longer necessary.

Notice that the API can be leveraged to enrich the interaction with the user. For example, a light could be used in addition the tablet to signal a notification. As is proposed by devices such as HUE by Philips, ${ }^{2}$ we could even use a different color to indicate whether the notification is non-critical or critical. Along the same line, notifications could be played to the user via a voice synthesizer. These choices allow to tailor the presentation of notification to the user's preferences, improving their satisfaction and adoption.

Finally, introducing an API allows to factorize the expertise in designing user interactions that are adapted to older adults. In particular, the format of the notification text is defined by default according to the standards used for older adults and disabled users (ISO 22411). ${ }^{3}$

\subsection{The Assisted Living Platform}

Our proposed approach is been carried out in practice in a platform for assisted living, dedicated to older adults. This platform is based on a range of building blocks, both hardware (e.g., motion

\footnotetext{
2 WWW . meet hue.com

${ }^{3}$ ISO/TR $22411-2008$.
}

detector, contact sensor, smart switch) and software (e.g., calendar, photo album, address book). In addition, the user is provided with a tablet, which is stationary, plugged, and located at a central location in the user's home. Interactions between the user and the assistive services revolve around this tablet. To prevent the tablet from stigmatizing the user, when it is idle, it becomes a digital frame, as shown in Figure 5. This service streams photos from a shared album (e.g., Picasa photo) that can be updated by family members and friends; it greatly contributes to the user acceptance and satisfaction of the platform.

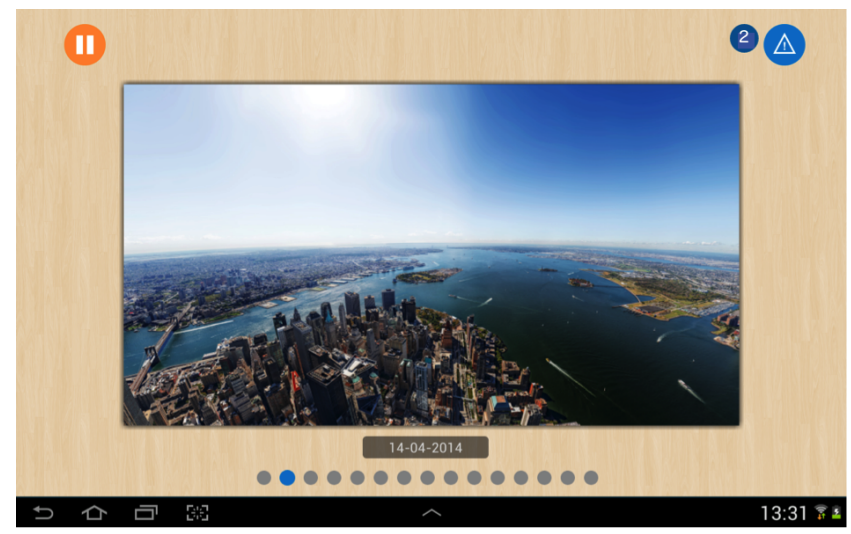

Figure 5: Digital frame

For the sake of completeness, note that the tablet provides the user with a control mechanism (top left corner of the screen) that allows to pause the platform at anytime for different durations.

\subsection{Deployed Services}

The assistive services of our field study cover the three domains of assisted living. Safety and security are addressed by two assistive services that monitor the entry door. During daytime, one service prompts the user when the door is open and left unattended for a given period of time. During nighttime, another service alerts the user when the door is opened. Both services use a critical interaction and solicit a caregiver if the user does not respond to the alert.

ADLs are covered by an application that reminds the user to perform a given set of activities (e.g., preparing a meal, dressing), when they have not been performed within a time window. This application leverages work on activity detection by Caroux et al. [5]. In this domain, another application sends reminders to the user prior to the due date of utility bills. Finally, a service monitors the fridge door to ensure it is not left open for too long.

Regarding social participation, an email notifier announces social events for older adults, organized by their municipality. Another service sends a notification when new email messages have been received by the user.

For the sake of completeness, note that other assistive services, beyond the scope of this work, are running in our participants' home. As mentioned earlier, when the tablet is idle, it turns into a digital photo frame. This service can be seen as stimulating social participation because family members and friends share their photos on this album. This digital frame does not require any interaction from the user. Another assistive service provided by our platform is a light path, to guide the user to the bathroom at night. This light path is controlled by the switch of a bed light. Here, we leverage an existing interaction to control the assistive service. 


\section{DESCRIPTION OF THE FIELD STUDY}

In this section, we present a field study aimed to validate our approach. We first evaluate the effectiveness and learnability of our notification system for older adults. Then, we assess the user experience by measuring user acceptance and satisfaction. This evaluation is based on the interactions triggered by the assistive services of our platform, equipping the homes of our study participants. From the user's perspective, the tablet serves service notifications and materializes the assisted living platform. Indeed, the hardware infrastructure of the platform consists of unobtrusive entities, such as motion detectors, contact sensors (battery powered), and smart switches; they are wireless and placed at discreet locations. Software entities are Web services accessible via Internet and only visible through the stimuli they generate for the platform (e.g., events). We provide empiral evidence that the notification system of our assisted living platform is suitable for older adults, even with cognitive and/or sensory loss.

Our assisted living platform was deployed in the house of 15 community-dwelling older adults (see below) for a 6-month period. Every six weeks, interaction data and questionnaires were collected to measure the longitudinal effect of the platform adoption. This data collection took place in the house of the participants and was performed by a research member of our group.

Note that the ecological nature of our field deployment requires a cautious approach considering that our participants are old older adults (81 years old on average). The assisted living platform manages potentially critical situations (e.g., door alert) that preclude the use of an unsuited notification system. Indeed, deploying an unsuited notification system to achieve a control condition for an experimental setup could have deleterious consequences for the participants [40]. This situation would rightfully raise serious concerns from an ethical committee.

\subsection{Participants}

We recruited 15 community-dwelling, old older adults. This was done in collaboration with a public home care service for older adults. We selected participants that live alone in their apartment or house. This choice addressed four key issues. First, this situation simplifies the design space of most assistive services (e.g., no usersensitive context). Second, assistive services can be customized with respect to a unique user, contributing further to user acceptance and satisfaction. Third, as reported in the literature [23], having multiple occupants in a home introduces sources of errors when monitoring activities. In turn, this situation has a negative effects on assistive services that are context sensitive, such as the ADL reminder. A final argument is that older adults living in couple are most of the time assisted by their spouse, and thus are less prompt to request for assistance.

As described in Figure 6, our participants were recruited according to specific exclusion criteria: dependency syndrome; neurological or musculoskeletal disease or systemic disorders. The main inclusion criterion was cognitive integrity with an MMSE (Mini Mental State Examination) [13] score greater than 24 [2]. According to the Helsinki declaration (WMA, 2008), approval was sought and obtained from the ethics committee of the University of Bordeaux. All participants provided a written consent form prior to the participation in our study.

We assessed their functional status for some activities of daily living. First, we evaluated their performance in IADLs, using the timed-IADL assessment test [29]. A participant is asked to perform an activity with a time limit. If the activity is achieved without error and without exceeding the time limit, a score of 1 is given. A score of 3 means that the participant has major difficulties to perform

\begin{tabular}{|c|c|}
\hline Participants & Mean $(S D)$ \\
\hline \hline Age & $81.07(6.19)$ \\
\hline Gender & 3 men and 12 women \\
\hline Education years & $9.60(1.55)$ \\
\hline Family status & 14 widowed and 1 single \\
\hline MMSE [0 - 30] & $27.80(1.37)$ \\
\hline Timed-IADL [5-15] & $5.40(0.74)$ \\
\hline Self-reported IADL [9-45] & $23.80(6.62)$ \\
\hline Cognitive resources $[0-162]$ & $149.60(7.90)$ \\
\hline Sensory resources $[0-4]$ & $2.67(0.95)$ \\
\hline
\end{tabular}

$S D=$ Standard Deviation .

Interval notations are used for score ranges.

\section{Figure 6: Participant profiles}

the activity. We tested our participants on five different activities; this gave scores ranging from 5 (ideal performance) to 15 (major difficulties). In Figure 6, we observe that our 15 participants obtain near ideal scores (5). We conclude that our participants show no difficulties in performing IADLs and have a high level of autonomy.

Then, we asked them to self-assess their functional status, using the 9-item IADL scale [19]. For each item, the participant assesses their performance: 1 denotes no difficulties and 5 denotes major difficulties. This tool shows that we have a variety of participants in the way they see themselves performing ADLs; it ranges from 15 to 36 , on a scale of 45 . In summary, our participants perform well in their ADLs, although they perceive themselves as experiencing difficulties. From these data, our participants exhibit a normal cognitive decline, along with complaints related to their everyday functioning.

Finally, to measure participants' cognitive resources, we summed two scores of widely used scales assessing cognitive functioning: the Dementia Rating Scale - 2 (DRS-2 ${ }^{4}$ ) [16] and the Frontal Assessment Battery $\left(\mathrm{FAB}^{5}\right)$ [10]. Sensory resources were measured using 2 items with 3 modalities (from 0 - poor abilities, to 2 - no impairment) regarding vision or audition.

\subsection{Timed usage scenarios}

Inspired by the timed-IADL assessment [29], we designed a test tool to measure the user's performance in using the tablet with two everyday usage scenarios, involving a critical interaction and a noncritical one. The two usage scenarios used are defined in Figure 7.

These two usage scenarios were selected from the deployed services because they are easy to implement. This test ranges on a scale of 3; the score depends on the type and number of errors made by the user, and whether the task was performed within the allocated time. A score of 3 denotes a task performed without errors and within the allocated time. A score of 2 corresponds to a task performed with minor errors but within the allocated time. A score of 1 is when the user did not complete the task or made major errors.

\subsection{Data Collected}

As mentioned earlier, we evaluate our approach by collecting measures of effectiveness and learnability of the notifications, as well as user acceptance and satisfaction of the assisted living platform. The period covered by our study starts six weeks after the platform is installed and ends after six month of using it, with data collected every six weeks (at 1.5, 3, 4.5 and 6 months of use).

\footnotetext{
${ }^{4}$ The DRS-2 measures five basic cognitive domains: attention, initiation, abstraction, verbal and non-verbal memory.

${ }^{5}$ The FAB assesses executive functioning, including controlled inhibition, flexibility and conceptualization.
} 


\begin{tabular}{|l|l|l|l|l|l|}
\hline Task & $\begin{array}{l}\text { Notification } \\
\text { type }\end{array}$ & Task conditions & Notification & $\begin{array}{l}\text { Instructions to the } \\
\text { user }\end{array}$ & Expected behavior \\
\hline \hline 1 & Critical & $\begin{array}{l}\text { The user is in front of the tablet; } \\
\text { the experimenter has left the entry } \\
\text { door open; a notification appears }\end{array}$ & $\begin{array}{l}\text { Alert: your door is } \\
\text { open }\end{array}$ & $\begin{array}{l}\text { "What is happening } \\
\text { and how should you } \\
\text { react?" }\end{array}$ & $\begin{array}{l}\text { "The door is open". The } \\
\text { user } \\
\text { button, goes to the entry } \\
\text { door and closes it. }\end{array}$ \\
\hline 2 & $\begin{array}{l}\text { Non- } \\
\text { critical }\end{array}$ & $\begin{array}{l}\text { The user is in front of the tablet; } \\
\text { the experimenter has scheduled } \\
\text { an appointment; a notification ap- } \\
\text { pears }\end{array}$ & $\begin{array}{l}\text { Reminder: Your } \\
\text { appointment to the } \\
\text { doctor is at 2:00pm }\end{array}$ & $\begin{array}{l}\text { "What is happening } \\
\text { and how should you have an appoint- } \\
\text { react?" }\end{array}$ & $\begin{array}{l}\text { 15s. } \\
\text { ment to my doctor } \\
\text { at 2:00pm"; the user } \\
\text { presses the OK button }\end{array}$ \\
\hline
\end{tabular}

Figure 7: Timed Usage Scenarios

Effectiveness and learnability. Our measure of effectiveness is the score given by the usage scenarios presented above. Our measure of learnability refers to the interaction duration to perform the task, measured in seconds. As mentioned earlier, we assessed effectiveness and learnability four times during our six-month study (every six weeks).

User acceptance. Our approach to measure user acceptance was inspired by an existing online tool proposed by Hassenzahl [15]. ${ }^{6}$ This measurement decomposes acceptance into five dimensions: ergonomic quality, hedonic quality, appealingness, anxiety and safety perception, and social influence. Answers to the questionnaire range over a scale of 7 points, from -3 to 3 , including two antonyms (e.g., nervous/relaxed). Each acceptance dimension of the questionnaire consists of 6 items. The global score (from -3 to 3 ) is computed by averaging the scores of the answers collected for the questionnaire. High acceptance is denoted by a high score.

Importantly, we did not use the Technology Acceptance Model (TAM) that stresses the perceived usefulness and perceived ease of use as the main attitudinal factors towards technology acceptance[8, 37]. Indeed, Chen and Chan conducted an experimental study involving 1012 seniors and demonstrated that technology acceptance is predicted by user characteristics, rather than attitudinal factors (perceived usefulness and ease of use) [7]. As a result, we decided to measure user acceptance beyond the two main attitudinal factors of TAM, including dimensions related to user experience using Hassenzahl's tool [15].

User satisfaction. We used the QUEST questionnaire [9] to measure user satisfaction. Each item of this questionnaire is evaluated on a scale ranging from 1 to 5 . A value 1 corresponds to "not satisfied at all' and a value 5 to 'very satisfied'. The questionnaire consists of 12 items and the total score is on a scale of 5. A high score denotes a high user satisfaction with the technology.

\section{ANALYSIS}

We now analyze the longitudinal measures of effectiveness and learnability over our 15 participants, for both critical and non-critical interactions with the tablet. Then, we analyze the measures of user acceptance and satisfaction. In addition, for each measure, we evaluate the impact of cognitive and sensory resources of the user to identify whether this system can effectively match the variability of the users in their declining abilities.

Effectiveness and learnability measures.

Thanks to two usage scenarios of the user managing critical and non-critical notifications, we assessed effectiveness and learnability of the platform. The performances of our participants are shown in Figure 8 for effectiveness and in Figure 9 for learnability. To

\footnotetext{
${ }^{6} \mathrm{http} / / /$ attrakdiff.de
}

study the evolution of these measures during the six-month period, we performed ANOVAs with the following statististical design: 2 independent factors [time of use (at 1.5, 3, 4.5 and 6 months) and notification type (critical vs. non-critical)], and effectiveness score or learnability time as dependent factors.

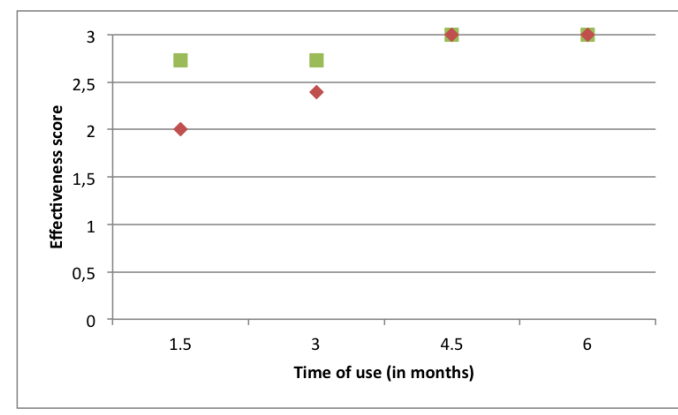

Effectiveness scores range from 0 to 3.

Diamond for critical notifications / Square for non-critical ones.

\section{Figure 8: Evolution of effectiveness}

First, we can observe a significant improvement of the effectiveness over the six-month period (effect of time: $\mathrm{F}(3.108)=8.11$; $p<0.001)$. Moreover, the ANOVA's results indicate a significant effect of the notification type $(\mathrm{F}(3.108)=5.74 ; \mathrm{p}=0.018)$, with higher values for non-critical notifications, implicating that scores are significantly higher for this notification type. No interaction between our two independent factors (i.e., time $\times$ notification type) was observed $(\mathrm{F}(3.108)=2.32 ; \mathrm{p}=0.080)$, which means that notification types do not evolve differently in a significant way over the period of the study.

Globally, these results indicate an improvement of effectiveness to answer both critical and non-critical notifications over the course of the study. A maximal performance is reached after 4.5 months of using our platform; this maximal performance remains over time.

Regarding learnability, we observe a significant improvement of the performance over time (effect of time: $\mathrm{F}(3.108)=10.45 ; p<$ $0.001)$, but no effect regarding the notification type $(\mathrm{F}(3.108)=$ $2.77 ; p=0.100)$. However, the ANOVA analysis indicates a significant interaction effect between the two independent factors (i.e., time $\times$ notification type $)(\mathrm{F}(3.108)=2.65 ; \mathrm{p}=0.05)$, which means that the learnability of critical and non-critical notifications evolves differently over time. More precisely, at six weeks of technology use, participants take more time to answer to a critical notification than a non-critical one. However, at six months of use, users perform equally well, irrespective of notification type. These results can be explained by an effective learning of the platform over time, 


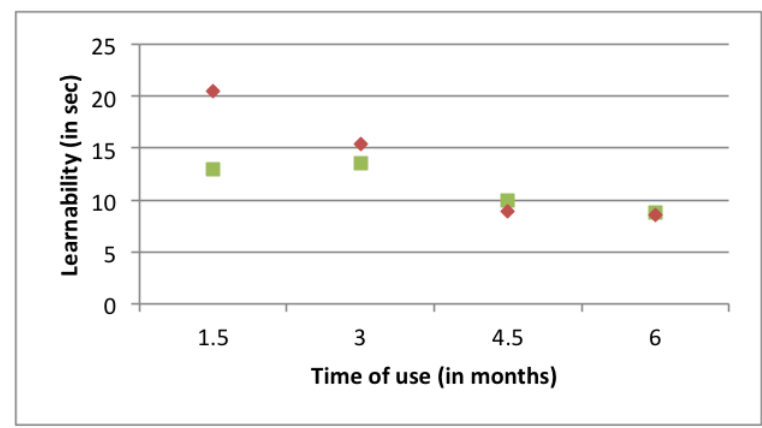

Time is measured in seconds.

Diamond for critical notifications / Square for non-critical ones.

\section{Figure 9: Evolution of learnability}

resulting in an automation of the process of answering critical notifications.

Finally, we performed two ANCOVAs with the following statistical design: time of use (at 1.5, 3, 4.5 and 6 months) and notification type (critical vs. non-critical) as independent factors, cognitive score (or sensory score) as continuous covariant factor, and effectiveness score or learnability time as dependent factors. Results highlight that cognitive and sensory resources have no impact on effectiveness and learnability. Thus, this platform is usable by older adults and its learnability is irrespective of cognitive or sensory loss.

Longitudinal assessment of user acceptance.

To measure user acceptance during the use of our platform, five dimensions of acceptance (ergonomic quality, hedonic quality, appealingness, anxiety and safety perception, and social influence) was assessed four times for our participants: 1) six weeks after the platform installation, 2) after 3 months of using the platform, 3) after 4.5 months, and 4 ) after 6 months. The longitudinal evolution of our five dimensions of acceptance was analyzed using MANOVA [5 dimensions (ergonomic quality, hedonic quality, appealingness, safety perception, and social influence) as dependent variables $\times$ 1 independent factor (time of measure)]. Figure 10 shows the acceptance scores given by our participants for theses measures, in addition to the mean score of these participants.

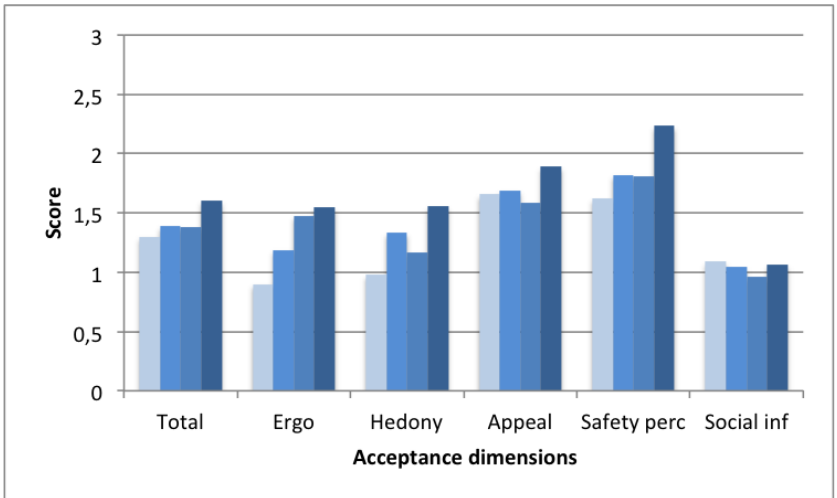

User acceptance scores from -3 to 3.

Four measures during the study (at 1.5, 3, 4.5 and 6 months) represented by the four shades of blue.

\section{Figure 10: Evolution of user acceptance}

Statistical analysis failed to be significative (all the effects with p $>0.200$ ), but we observe that high scores are obtained over the course of the study, with an improvement for the majority of vari- ables, mainly for the ergonomic and safety perception dimensions. The global acceptance score shows a slight increase too.

Then, in order to investigate the implication of cognitive and sensory resources on technology acceptance, we performed ANCOVAs with the following statistical design: time (at 1.5, 3, 4.5 and 6 months) as independent factor, acceptance dimensions as dependent factor, and cognitive score (or sensory score) as continuous covariant factor (Figure11). When a significant effect was found, we used inter-correlation analysis to identify the correlation (positive or negative) between the factors.

\begin{tabular}{lcc}
\hline & $\begin{array}{c}\text { Cognitive } \\
\text { resources }\end{array}$ & $\begin{array}{c}\text { Sensory } \\
\text { Resources }\end{array}$ \\
\hline Global acceptance & $\mathbf{F}(\mathbf{3 . 5 3})=\mathbf{4 . 6 8} *$ & $\mathbf{F}(\mathbf{3 . 5 3})=\mathbf{4 . 0 4} *$ \\
Ergonomy & $\mathbf{F}(\mathbf{3 . 5 3})=\mathbf{4 . 0 2} *$ & $\mathrm{~ns}$ \\
Hedony & $\mathbf{F}(\mathbf{3 . 5 3})=\mathbf{5 . 7 0 *}$ & $\mathbf{F}(\mathbf{3 . 5 3})=\mathbf{3 . 9 7} \ddagger$ \\
Appealingness & $\mathbf{F}(\mathbf{3 . 5 3})=\mathbf{3 . 3 2} \ddagger$ & $\mathbf{F}(\mathbf{3 . 5 3})=\mathbf{4 . 3 1 *}$ \\
Safety perception & $\mathrm{ns}$ & $\mathrm{ns}$ \\
Social influence & $\mathbf{F}(\mathbf{3 . 5 3})=\mathbf{3 . 2 4} \ddagger$ & $\mathbf{F}(\mathbf{3 . 5 3})=\mathbf{4 . 6 8 *}$ \\
\hline
\end{tabular}

ANCOVAs=analysis of covariance

${ }^{*} p<0.05 ; \ddagger p<0.08 ;$ ns=non significant .

Figure 11: ANCOVAs results controlling for cognitive or sensory scores

Results indicate a significant effect of the cognitive resources of the users on most of the acceptance dimensions (every variable except safety perception). Inter-correlations show negative relation between these factors (all the $r<-0.22$; all the $p<0.07$ ), indicating that the more cognitive resources are deteriorated, the more technology is accepted. Regarding sensory resources, we can observe a significant relationship with some dimensions (hedony, appealingness, social influence, and the total score of acceptance), with a positive correlation (all the $r>0.25$; all the $p<0.05$ ). This result implies that the more sensory aptitudes are diminished, the more technology acceptance is low.

Globally, these results highlight the constant improvement of user acceptance, showing that users are comfortable with the technology and confident that they can handle their interactions with it. Furthermore, we observed a better acceptance when users have poor cognitive resources and mild sensory decline, showing that the system does not require many resources to interact with it.

User satisfaction.

We administered the QUEST questionnaire to measure user satisfaction about the technology after 1.5, 3, 4.5 and 6 months of usage experience.

We observed that the satisfaction score was already high for all of our participants after 1.5 month of use (the mean score is 4.28 for a max score of 5), and did not statistically differ over time (ANOVA: $\mathrm{F}(3.53)=0.41 ; \mathrm{p}=0.75)$.

By doing the same statistical design as above (i.e., ANCOVAs with cognitive and sensory resources as covariant), we only observe a significant effect of cognitive resources on satisfaction score $(\mathrm{F}(3.53)=4.68 ; \mathrm{p}=0.035)$, with a negative correlation $(\mathrm{r}=-0.29 ; \mathrm{p}$ $=0.03$ ), indicating that users with low cognitive resources are more satisfied with the technology.

Overall, participants exhibit a high satisfaction score regarding our technology, and this satisfaction remains constant over a period of six months. Moreover, this satisfaction score is further enhanced for users with poor cognitive aptitudes, and remains unchanged for user with declining sensory resources. 


\section{DISCUSSION}

This paper presents our approach to scaling up assistive services by homogenizing notifications. We measured during a six-month study the evolution of the effectiveness, learnability, and acceptance of our platform embedded in the daily life of fifteen older adults. Moreover, we assessed the relation of such variables as users' abilities (i.e., cognitive and sensory resources).

Our results indicate that unifying notifications allows the user to be fully acquainted with the interactions of our platform. This acquaintance improves over the study period, in terms of effectiveness, learnability, and acceptance.

Regarding effectiveness and learnability, we observe an effective acquaintance with the platform, irrespective of the cognitive and sensory resources of the users. This means that this platform is suitable for the interaction abilities of older adults. Interestingly, the learnability performance reveals that the time to be-performed task for critical notification condition is longer than the time for non-critical condition during the first period of technology use (1.5 months). Thus, the older participants have given more processing time for critical notifications as expected, considering the potential consequence for the participant. In other words, the critical and noncritical notifications are accurately distinguished by the participants and thus, they are processed according to their nature [22]. The increasing practice of the system allows the user to automate the process, resulting in an equivalent handling time for both notification types, after three months. This is even more evident when considering that critical notifications require more time to be handled by the participants since an action has be taken in the environment.

Furthermore, our results highlight a better acceptance for users with poor cognitive resources, particulary regarding ergonomy and hedonism. Thus, it seems that this platform effectively match older users' needs by offering services assisting cognitive decline [12] (e.g., entry door monitoring or appointment reminders).

Concerning sensory abilities, we observe a lower acceptance for users with diminished sensory abilities, particulary for the dimensions of appealingness and social influence. This result could be explained by the fact that our platform use multi-modal notifications (i.e., tone, text, specifc colors and shapes). This approach could discourage users with a visual and/or auditive decline from adopting the technology

To the best of our knowledge, there is no study aiming at scaling up assistive services by unifying notifications. Furthermore, there is no study measuring the effectiveness and learnability of a user interface that operates a range of assistive services in an ecological setting. Such study provides insights on how to increase the number of assistive services, without increasing their cognitive cost. More globally, our work provides some of the first empirical evidence that an assisted living platform can range over the domains of assisted living, without overwhelming older adult users [34], as shown by our results on user acceptance and satisfaction.

The design of our notification system has leveraged the literature on notifications (e.g., $[36,26])$ and the design principles and guidelines for technology when targeting older adults [12]. As well, we have taken into account the specificities of older adults related to their susceptibility to stimuli [40], the cognitive cost of technology [7], and a suitable model to measure their acceptance of technology $[15,7]$.

\subsection{Limitations}

Notifications are simple and may appear as lacking expressiveness. An analogy could be drawn between these notifications and text messages on a cell phone. Although this analogy oversimplifies our approach, it captures the idea that we provide a way for a variety of assistive services to deliver uniform messages to the user. This is a powerful medium to support independent living of a declining older adult without overwhelming them. Still, we are continuing the analysis of existing and new services to determine how wide a range of assistive services can be captured by our notion of notifications, and whether and how they could be enriched without incurring a cognitive cost.

Task-specific interactions are not studied. Our goal was to concentrate on the interactions that matched a common pattern, which was recurring in the usage of assistive services. Our participants accepted the way interactions of assistive services are structured as a result of our proposed decomposition. In any case, we plan to further analyze task-specific interactions using domain engineering, from software engineering, by identifying their commonalities and variabilities.

Notifications are only implemented for tablets. We have not yet explored our approach beyond tablets. In our platform, this device is stationary and always on, ready to deliver notifications. This may have been reassuring for our participants because when hearing the tone of a notification, they knew where to go to handle it. Using a $\mathrm{TV}$ is more difficult because it is not always one. If the platform were able to turn it on, it could be disturbing for the user.

\subsection{Perspectives}

Towards a methodology to design notifications for older adults. When developing assistive services, we examined existing assistive systems and the wide range of design strategies adopted by other developers regarding notifications. When notifications are recurring, this diversity gets the user confused or/and frustrated. Our approach is a step towards enabling the developer of an assistive service to use our approach as a methodology to define the notifications. The API offers a layer that abstracts over some of the implementation requirements of interacting with an older adult users (auditory, visual). We want to explore this issue further. In particular, we would like to conduct a study that consists of comparing the development of assistive services, with and without our approach.

Enriching notifications. When analyzing existing assistive services, we identified a need for a notification that would inform the user about the progression of a task being performed. This could be used in multi-step tasks, such as taking pills, personal care, and meal preparation. Typically, this information could be delivered visually as a progress bar. The application monitoring the task could use an extended API to provide the user with a feedback, whenever a notification with the environment is detected via sensors.

Leveraging the simplicity of notifications.. There is a range of users that could benefit from the simplicity and homogeneity of notifications. This includes users with intellectual deficiencies, age-related neuro-degenerative diseases (e.g., Alzheimer, Parkinson) and neurological disorders (e.g., traumatic brain injuries, cerebral vascular diseases). In the coming months, we are initiating a project aimed at providing an assisted living platform for adults with Down syndrome.

\section{CONCLUSIONS}

In this paper, we presented an approach to scaling up assistive services and address the increasingly many needs of older adults with cognitive decline. Our approach consists of decomposing the user interactions of assistive services into two categories: notifications and task specific. We observe that notifications are often the 
recurring interactions and they can be homogenized to lower the cognitive cost of assistive services. We propose an API to implement notifications in assistive services. We validate our approach by applying it to a range of existing and new assistive services.

Our approach is implemented in an assisted living platform. Notifications are supported by a tablet. This platform has been deployed at the home of 15 older adults. A six-month study has shown the effectiveness and learnability of the notification system of our platform, irrespective of the cognitive and sensory resources of the users. Additional results showed that our assisted living platform has achieved high user acceptance and satisfaction.

\section{ACKNOWLEDGMENTS}

We thank the members of the Phoenix research group at Inria for their contributions to this project. We also thank the anonymous reviewers for their thorough reviews that improved our paper significantly. Our project is funded by the following sponsors: UDCCAS, CG33, CRA, CNSA, Chambre des métiers.

\section{REFERENCES}

[1] Abowd, G. D., and Mynatt, E. D. Designing for the human experience in smart environments. Smart environments: technologies, protocols, and applications (2004), 151-174.

[2] Albert, M. S., DeKosky, S. T., Dickson, D., Dubois, B., Feldman, H. H., Fox, N. C., Gamst, A., Holtzman, D. M., Jagust, W. J., Petersen, R. C., et al. The diagnosis of mild cognitive impairment due to alzheimer's disease: Recommendations from the national institute on aging-alzheimer's association workgroups on diagnostic guidelines for alzheimer's disease. Alzheimer's \& Dementia 7, 3 (2011), 270-279.

[3] Bouisson, J. Routinization preferences, anxiety, and depression in an elderly french sample. Journal of Aging Studies 16, 3 (2002), 295-302.

[4] Caine, K. E., Zimmerman, C. Y., Schall-Zimmerman, Z., Hazlewood, W. R., Sulgrove, A. C., Camp, L. J., Connelly, K. H., Huber, L. L., and Shankar, K. Digiswitch: design and evaluation of a device for older adults to preserve privacy while monitoring health at home. In Proceedings of the 1st ACM International Health Informatics Symposium, ACM (2010), 153-162.

[5] Caroux, L., Consel, C., Dupuy, L., and Sauzéon, H. Verification of Daily Activities of Elders: A Simple, Non-Intrusive, Low-Cost Approach. In ASSETS - The 16th International ACM SIGACCESS Conference on Computers and Accessibility (Rochester, NY, United States, 2014).

[6] Chan, M., Estève, D., Escriba, C., and Campo, E. A review of smart homes- present state and future challenges. Computer methods and programs in biomedicine 91, 1 (July 2008), 55-81.

[7] Chen, K., and Chan, A. H. S. Gerontechnology acceptance by elderly Hong Kong Chinese: a senior technology acceptance model (STAM). Ergonomics 57, 5 (2014), 635-652.

[8] Davis, F. D., Bagozzi, R. P., and Warshaw, P. R. User acceptance of computer technology: a comparison of two theoretical models. Management science 35, 8 (1989), 982-1003.

[9] Demers, L., Monette, M., Lapierre, Y., Arnold, D., and Wolfson, C. Reliability, validity, and applicability of the Quebec User Evaluation of Satisfaction with assistive Technology (QUEST 2.0) for adults with multiple sclerosis. Disability \& Rehabilitation 24, 1-3 (2002), 21-30.
[10] Dubois, B., Slachevsky, A., Litvan, I., and Pillon, B. The FAB: A frontal assessment battery at bedside. Neurology 55, 11 (2000), 1621-1626.

[11] Findlater, L., Froehlich, J. E., Fattal, K., Wobbrock, J. O., and Dastyar, T. Age-related differences in performance with touchscreens compared to traditional mouse input. In Proceedings of the SIGCHI Conference on Human Factors in Computing Systems, ACM (2013), 343-346.

[12] Fisk, A. D., Rogers, W. A., Charness, N., Czaja, S. J., and Sharit, J. Designing for older adults: Principles and creative human factors approaches. CRC press, 2012.

[13] Folstein, M. F., Folstein, S. E., and McHugh, P. R. Mini-mental state: a practical method for grading the cognitive state of patients for the clinician. Journal of psychiatric research 12, 3 (1975), 189-198.

[14] Gillespie, A., Best, C., and O’Neill, B. Cognitive function and assistive technology for cognition: a systematic review. Journal of the International Neuropsychological Society : JINS 18, 1 (Jan. 2012), 1-19.

[15] Hassenzahl, M. The interplay of beauty, goodness, and usability in interactive products. Human-Computer Interaction 19, 4 (2004), 319-349.

[16] Jurica, P. J., Leitten, C. L., and Mattis, S. DRS-2 dementia rating scale-2: professional manual. Psychological Assessment Resources, 2004.

[17] Khan, D. U., Siek, K. A., Meyers, J., Haverhals, L. M., Cali, S., and Ross, S. E. Designing a personal health application for older adults to manage medications. In Proceedings of the 1st ACM International Health Informatics Symposium, ACM (2010), 849-858.

[18] Laurienti, P. J., Kraft, R. A., Maldjian, J. A., Burdette, J. H., and Wallace, M. T. Semantic congruence is a critical factor in multisensory behavioral performance. Experimental brain research 158, 4 (2004), 405-414.

[19] Lawton, M. P., Moss, M., Fulcomer, M., and Kleban, M. H. A research and service oriented multilevel assessment instrument. Journal of Gerontology 37, 1 (1982), 91-99.

[20] Lee, M. L., and Dey, A. K. Reflecting on pills and phone use: supporting awareness of functional abilities for older adults. In Proceedings of the SIGCHI Conference on Human Factors in Computing Systems, ACM (2011), 2095-2104.

[21] Lee, M. L., and Dey, A. K. Real-time feedback for improving medication taking. In Proceedings of the 32nd annual ACM conference on Human factors in computing systems, ACM (2014), 2259-2268.

[22] Lindenberger, U., Lövdén, M., Schellenbach, M., Li, S.-C., and Krüger, A. Psychological principles of successful aging technologies: A mini-review. Gerontology 54, 1 (2008), 59-68.

[23] Logan, B., Healey, J., Philipose, M., Tapia, E. M., and Intille, S. A Long-Term Evaluation of Sensing Modalities for Activity Recognition. In Ubicomp, Springer-Verlag, Ed. (Berlin Heidelberg, 2007).

[24] McCrickard, D. S., Catrambone, R., Chewar, C. M., and Stasko, J. T. Establishing tradeoffs that leverage attention for utility: empirically evaluating information display in notification systems. International Journal of Human-Computer Studies 58, 5 (2003), 547-582.

[25] McCrickard, D. S., Chewar, C. M., Somervell, J. P., and Ndiwalana, A. A model for notification systems evaluation: assessing user goals for multitasking activity. $A C M$ 
Transactions on Computer-Human Interaction (TOCHI) 10, 4 (2003), 312-338.

[26] McCrickard, D. S., Czerwinski, M., and Bartram, L. Introduction: design and evaluation of notification user interfaces. International Journal of Human-Computer Studies 58, 5 (2003), 509-514.

[27] Mokhtari, M., Aloulou, H., Tiberghien, T., Biswas, J., Racoceanu, D., and Yap, P. New trends to support independence in persons with mild dementia - a mini-review. Gerontology 58, 6 (2012), 554-563.

[28] Mozolic, J., Hugenschmidt, C., Peiffer, A., and Laurienti, P. Multisensory integration and aging. CRC Press, Boca Raton (FL) (2012).

[29] Owsley, C., Sloane, M., McGwin Jr, G., and Ball, K. Timed instrumental activities of daily living tasks: Relationship to cognitive function and everyday performance assessments in older adults. Gerontology 48, 4 (2002), 254-265.

[30] Rantz, M. J., Skubic, M., Koopman, R. J., Phillips, L., Alexander, G. L., Miller, S. J., and Guevara, R. D. Using sensor networks to detect urinary tract infections in older adults. In e-Health Networking Applications and Services (Healthcom), 2011 13th IEEE International Conference on, IEEE (2011), 142-149.

[31] Rashidi, P., and Mihailidis, A. A Survey on Ambient-Assisted Living Tools for Older Adults. IEEE Journal of Biomedical and Health Informatics 17, 3 (May 2013), 579-590.

[32] Riche, Y., and Mackay, W. PeerCare: Supporting Awareness of Rhythms and Routines for Better Aging in Place. Computer Supported Cooperative Work (CSCW) 19, 1 (Nov. 2009), 73-104.

[33] Rowan, J., and Mynatt, E. D. Digital family portrait field trial: Support for aging in place. In Proceedings of the SIGCHI conference on Human factors in computing systems, ACM (2005), 521-530.

[34] Scherer, M. J. Assistive technologies and other supports for people with brain impairment. Springer Publishing Company, 2012.

[35] Vastenburg, M. H., Keyson, D. V., and De Ridder, H. Considerate home notification systems: a field study of acceptability of notifications in the home. Personal and Ubiquitous Computing 12, 8 (2008), 555-566.

[36] Vastenburg, M. H., Keyson, D. V., and De Ridder, H. Considerate home notification systems: A user study of acceptability of notifications in a living-room laboratory. International Journal of Human-Computer Studies 67, 9 (2009), 814-826.

[37] Venkatesh, V., Morris, M. G., Davis, G. B., and Davis, F. D. User acceptance of information technology: Toward a unified view. MIS quarterly (2003), 425-478.

[38] Warnock, D., McGee-Lennon, M., and Brewster, S. The role of modality in notification performance. In Human-Computer Interaction-INTERACT 2011. Springer, 2011, 572-588.

[39] Warnock, D., McGee-Lennon, M., and Brewster, S. Multiple notification modalities and older users. In Proceedings of the SIGCHI Conference on Human Factors in Computing Systems, ACM (2013), 1091-1094.

[40] Wetherell, J. L., Reynolds, C. A., Gatz, M., and Pedersen, N. L. Anxiety, cognitive performance, and cognitive decline in normal aging. The Journals of Gerontology Series B: Psychological Sciences and Social Sciences 57, 3 (2002), P246-P255.

[41] Wiley, J., Sung, J.-Y., and Abowd, G. The Message Center : Enhancing Elder Communication. In CHI 2006 (Montreal, Canada, 2006), 1523-1528. 